CASE REPORT

\title{
Collagenous spherulosis in an adenomyoepithelioma of the breast
}

\author{
J S Reis-Filho, L G Fulford, B Crebassa, S Carpentier, S R Lakhani
}

J Clin Pathol 2004;57:83-86

Collagenous spherulosis (CS) is an incidental finding that usually occurs in association with benign proliferative and preinvasive lesions. Adenomyoepithelioma is a biphasic neoplasm of the breast, composed of both luminal epithelial and myoepithelial cells. Despite the common epimyoepithelial histogenesis, CS has never been described in association with adenomyoepithelioma. This report describes the case of a 48 year old woman who presented with CS of the breast in an adenomyoepithelioma. The combination of these two benign lesions led to diagnostic difficulties; namely, differentiation from adenoid cystic carcinoma, but also cribriform carcinoma and cylindroma of the breast. Antibodies to the oestrogen receptor, progesterone receptor, p63, and c-kit (CD1 17) proved to be useful adjuncts to differentiate between these lesions.

$\mathrm{T}$ he patient was a 48 year old woman who was undergoing regular mammographic surveillance following detection of microcalcifications in the right breast in 1987. Biopsy at that time revealed atypical ductal hyperplasia and she had remained well since. Family history was unremarkable. On the most recent mammogram, a retroareolar, non-palpable nodule was detected in the left breast. The lesion was excised and submitted for pathological examination. No further treatment was carried out. The patient remains free of disease six months after the diagnosis and continues to be followed up.

\section{PATHOLOGICAL FINDINGS}

Gross examination revealed a well circumscribed, whitish, solid nodule, measuring $6 \mathrm{~mm}$ in greatest diameter. Microscopic evaluation disclosed a well circumscribed but non-encapsulated solid lesion (fig 1A) composed of abortive dual layered gland-like structures and nests of faint eosinophilic and clear myoepithelial cells arranged around compressed and elongated epithelial lined lumina, some of which showed papillary growth patterns and apocrine metaplasia. The stroma was composed of hyalinised collagenous septae of varying thickness, which divided the lesion into nests and lobules of different sizes and shapes (fig 1B). Occasional solid areas exclusively composed of myoepithelial cells were also found. The overall mitotic index was two mitoses/10 high power fields (HPFs). The histological findings were typical of a lobulated adenomyepithelioma.

Focally, at the periphery of the tumour there was an area of differing morphology (fig lA, C). Here, nests composed of small myoepithelial-like cells containing oval nuclei and ill defined cuboidal to spindle shaped cytoplasm were arranged around focal aggregates of well circumscribed, deeply eosinophilic periodic acid Schiff D positive acellular spherules (fig 1D, E). These nests imperceptibly merged with the typical adenomyoepitheliomatous areas (fig $1 \mathrm{C}$ ). The mitotic index of these areas was two mitoses/10 HPFs.

Table 1 summarises the immunohistochemical analysis. Myoepithelial cells were strongly positive for $\alpha$ smooth muscle actin, S-100 protein, and p63; focally positive for calponin, smooth muscle myosin heavy chain (SMMHC), cytokeratin 5/6 (CK5/6), and CK14; and negative for CK19, c-kit (CDI17), the oestrogen receptor (ER), and the progesterone receptor (PR). As expected, the myoepithelial cells of adjacent breast lobules and ducts did not express the last four markers, whereas luminal epithelial cells of the lesion and adjacent luminal epithelial cells were positive for CK19, c-kit, ER, and PR. In the peripheral areas, the cells arranged around the acellular spherules showed strong p63 reactivity (fig $2 \mathrm{C}$ ), in addition to focal expression of calponin, SMMHC, and CK14, indicating their myoepithelial nature. Focal CK19, ER (fig 2A), and PR (fig 2B) expression was also seen. The basal membranes around the cell nests and spherules were positive for collagen IV (fig IE). Importantly, c-kit was uniformly negative in these areas (fig 2D). There were no significant differences between the Ki67 labelling indices in the central and peripheral areas.

Hence, based on the histological features and immunohistochemical profile, a diagnosis of adenomyoepithelioma (AME) with admixed collagenous spherulosis (CS) at the periphery was made.

\section{DISCUSSION}

AME is a solitary, well circumscribed neoplasm characterised by a biphasic proliferation of both luminal epithelial and myoepithelial cells, ${ }^{1-3}$ which can be subclassified into spindle cell, tubular, and lobulated types according to the growth pattern and cell morphology. ${ }^{2}$ Most AMEs behave in a benign fashion, ${ }^{1-3}$ although cases of malignant AME and carcinomas arising in AME have also been described. ${ }^{1-3}$

CS is a fairly uncommon benign lesion composed of a mixture of epithelial and myoepithelial cells. ${ }^{45}$ It is usually multifocal and an incidental finding in breast samples removed for other reasons. ${ }^{45}$ CS has been described in association with various benign and malignant lesions, including sclerosing adenosis, radial scar, intraductal papilloma, fibroadenoma, atypical ductal hyperplasia, ductal carcinoma in situ, and lobular carcinoma in situ. ${ }^{45}$ Not surprisingly, in view of the structural similarities to breast, CS has been seen in salivary gland and cutaneous myoepithelial tumours. Of note, the diagnosis of CS in breast biopsies has been a major source of confusion. ${ }^{45}$ In the AFIP

Abbreviations: $\mathrm{AdCC}$, adenoid cystic carcinoma; $\mathrm{AME}$, adenomyoepithelioma; CS, collagenous spherulosis; ER, oestrogen receptor; HPF, high power field; PR, progesterone receptor; SMMHC, smooth muscle myosin heavy chain 


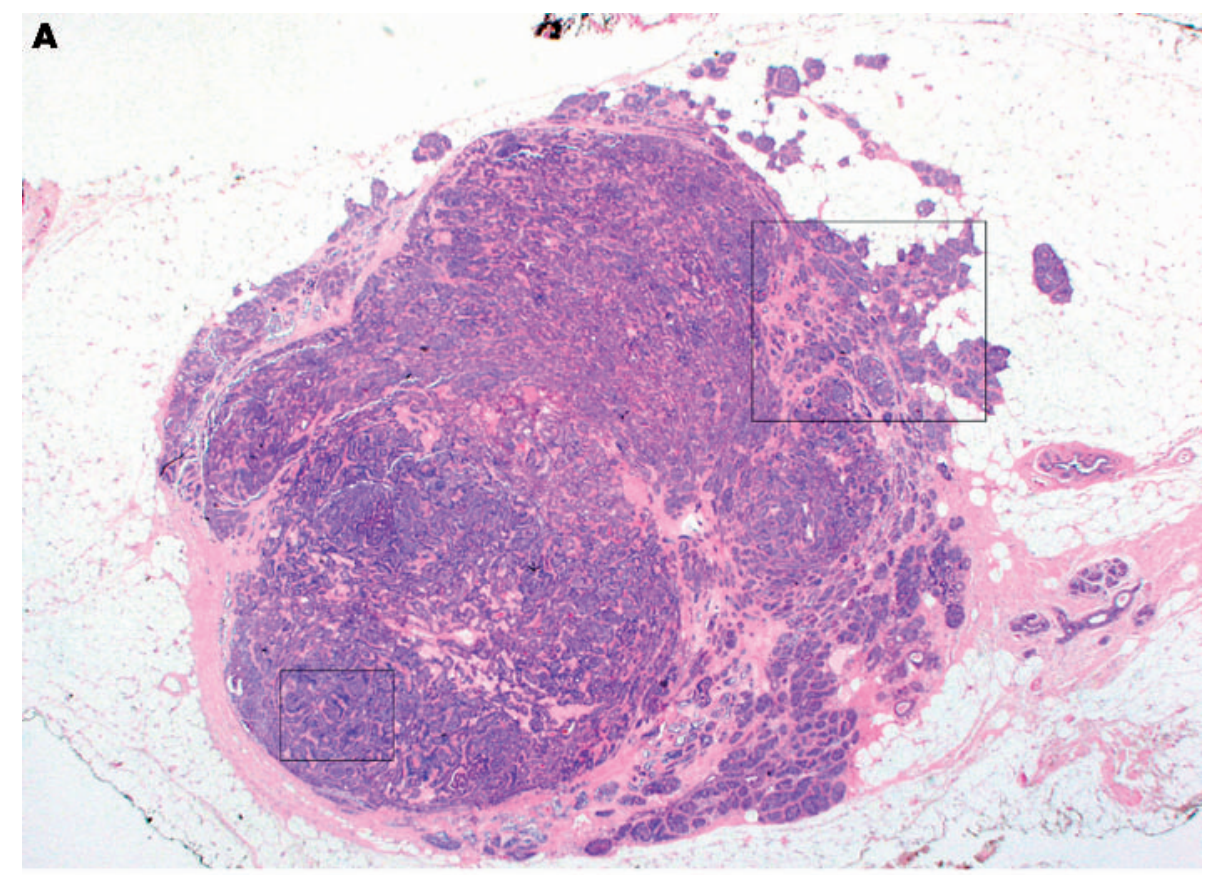

Figure 1 Adenomyoepithelioma with collagenous spherulosis. (A) Low power view disclosing the well circumscribed and non-encapsulated nature of the lesion. (B) Areas showing typical histological features of lobulated adenomyoepithelioma. (C, D) Details of the nests, which were composed of luminal and myoepithelial cells, arranged around focal aggregates of well circumscribed, deeply eosinophilic acellular spherules. These nests imperceptibly merged with the typical adenomyoepitheliomatous areas (C, arrow). (E) Detail of the peripheral areas disclosing luminal epithelial and myoepithelial cells arranged around the collagen IV positive acellular spherules.
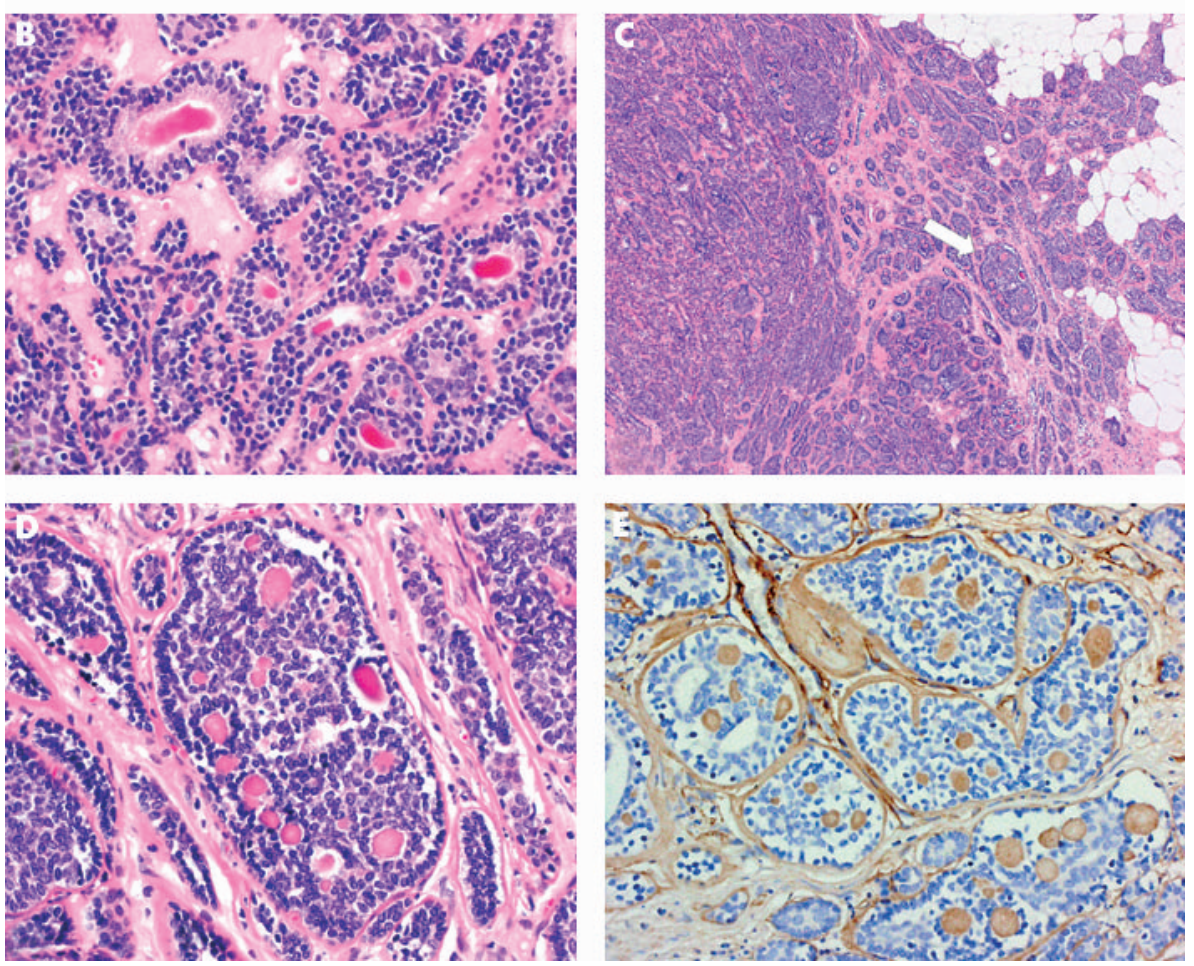

series, ${ }^{5}$ on review of 81 cases of CS sent for second opinion, only $15 \%$ had been correctly diagnosed by the referring pathologist. Most were either overlooked or misdiagnosed as atypical ductal hyperplasia, cribriform ductal carcinoma in situ, secretory carcinoma, mucinous carcinoma, or adenoid cystic carcinoma (AdCC). ${ }^{5}$

Intriguingly, despite the histogenetic similarities between AME and CS, to the best of our knowledge no cases of CS in AME of the breast have been reported to date. In our study, we report a previously unrecognised association of these two epimyoepithelial lesions. The differential diagnoses in our present case included adenoid cystic ${ }^{6-8}$ or cribriform ${ }^{5}$ carcinoma arising in AME, and cylindroma (dermal analogue tumour $)^{9}$ of the breast.
"The diagnosis of collagenous spherulosis in breast biopsies has been a major source of confusion"

AdCC of the breast is a rare, special type of invasive breast carcinoma, accounting for $0.05-0.10 \%$ of all primary carcinomas of the breast. ${ }^{6-8}$ In contrast to its salivary gland counterpart, breast AdCC is usually macroscopically well demarcated, but infiltrating and occasionally multifocal. ${ }^{6}$ AdCC may have a plethora of histological patterns; however, the presence of cribriform cylindromatous areas is mandatory for its diagnosis. ${ }^{6}{ }^{7}$ Briefly, these areas are characterised by a varying proportion of small "basaloid" myoepithelial cells and luminal epithelial cells arranged in cribriform gland-like spaces filled with homogeneous, amorphous, eosinophilic, or 

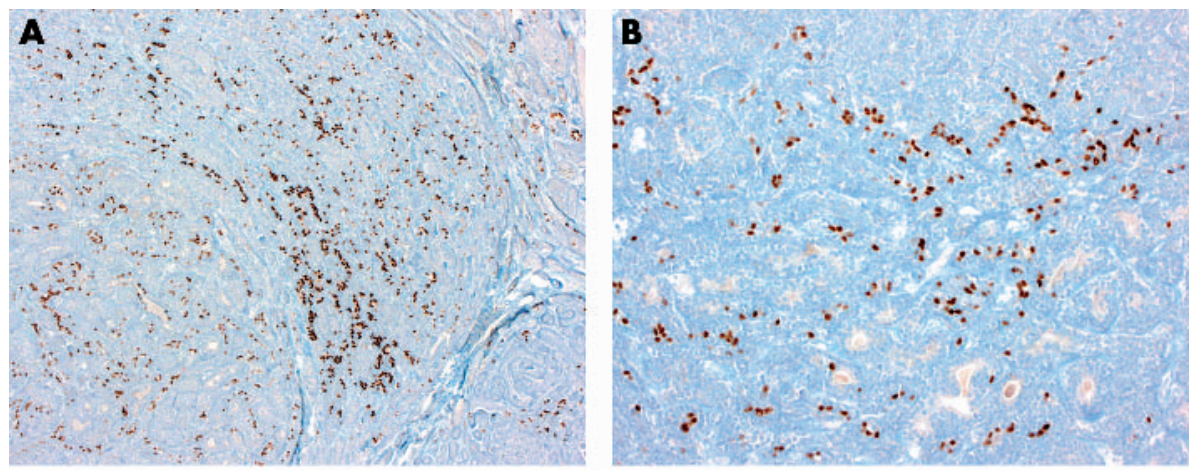

Figure 2 Immunohistochemical findings. (A) Oestrogen receptor. (B) Progesterone receptor. (C) p63 expression in the areas of collagenous spherulosis. Inset: note that the cells lining the collagen spherules are strongly positive for p63. (D) Lack of c-kit (CD1 17) expression in the collagenous spherulosis in contrast to the strong positivity in adjacent normal luminal cells (arrow).
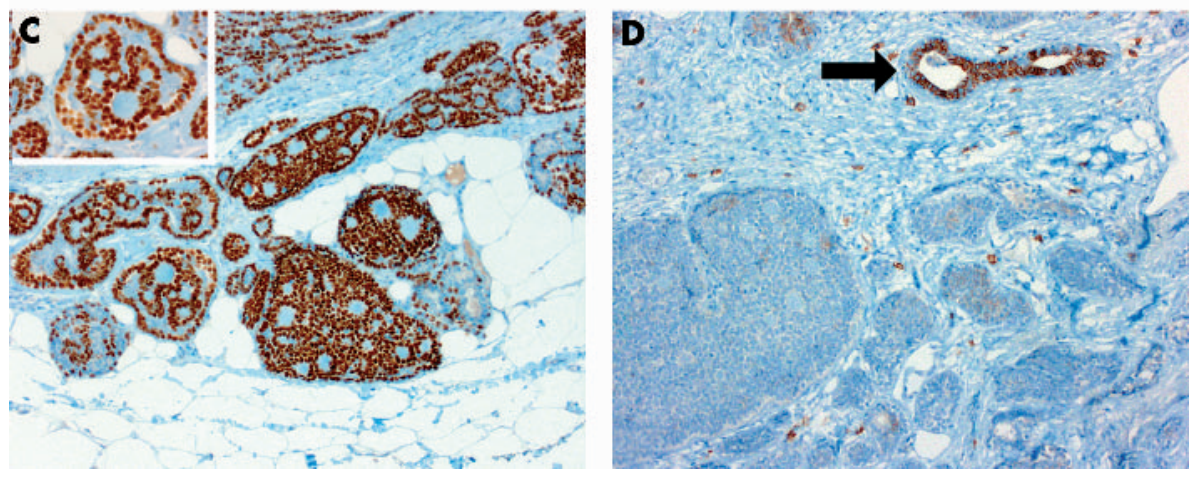

slightly basophilic material, immersed in myxohyaline stroma. ${ }^{6}$ Some tumours may be exclusively composed of basaloid cells. Areas of squamous and sebaceous differentiation may also be seen. ${ }^{67}$ At the immunohistochemical level, AdCCs consistently express high molecular weight CKs (34ßE12, 5/6, and 14), p63, glial fibrillary protein, S-100 protein, and calponin in the myoepithelial cells, whereas luminal cells react with antibodies against CK7 and CK19. ${ }^{78}$ ER reactivity in AdCC has been reported in $0-47 \%$ of cases, with positivity being dependant on the selection of cases, methods of fixation, and detection. ${ }^{67}$ Trendell-Smith and colleagues $^{7}$ emphasised that lack of ER and PR, as assessed by immunohistochemistry on paraffin wax embedded tissue, may be used to confirm a diagnosis of AdCC of the breast, because all of the bona fide cases of AdCC reported to date are consistently negative for ER and PR using this technique. ${ }^{7}$ Recently, the c-kit oncogene has received increased attention because of its biological, diagnostic, and prognostic implications for gastrointestinal stromal tumours. Interestingly, luminal cells of breast and salivary glands consistently express this tyrosine kinase receptor and it is also differentially expressed in salivary gland and breast neoplasms. ${ }^{10}$ Despite the lack of activating mutations of the KIT gene, ${ }^{10}$ c-kit is consistently expressed in most adenoid cystic carcinomas. $^{10}$

Two cases of AdCC arising in a tubular and a lobulated AME have been described. ${ }^{8}$ Both the reported cases showed features at low magnification that resembled those seen in our present case. The diagnosis of CS instead of AdCC was made in our present case because of the lack of capsule or stromal/fat invasion; the absence of small epithelium lined, duct-like spaces admixed with larger myoepithelial lined pseudocysts and extracellular mucoid secretory material; the presence of myoepithelial cells surrounding the acellular

Table 1 Immunohistochemical findings

\begin{tabular}{|c|c|c|c|c|c|c|c|}
\hline \multirow[b]{2}{*}{ Antiserum } & \multirow[b]{2}{*}{ Source } & \multirow[b]{2}{*}{ Clone } & \multirow[b]{2}{*}{ Dilution } & \multicolumn{2}{|l|}{ AME } & \multicolumn{2}{|l|}{ CS } \\
\hline & & & & LECs & MECs & LECs & MECs \\
\hline CK19 & Dako, Ely, Cambridgeshire, UK & RCK108 & $1 / 40$ & ++ & - & + & - \\
\hline CK14 & Novocastra, Newcastle, UK & LLOO2 & $1 / 40$ & - & ++ & + & - \\
\hline CK5/6 & Chemicon, Chandler's Ford, Hampshire, UK & D5/16B4 & $1 / 600$ & + & ++ & - & + \\
\hline S-100 & Dako & Polyclonal & $1 / 6000$ & + & ++ & + & + \\
\hline$\alpha$-SMA & Dako & lA4 & $1 / 150$ & - & ++ & - & + \\
\hline Calponin & Biogenex, San Ramon, California, USA & CALP & $1 / 3000$ & - & ++ & - & + \\
\hline SMMHC & Dako & SMMS-1 & $1 / 100$ & - & ++ & - & + \\
\hline p63 & $\begin{array}{l}\text { Santa Cruz Biotechnology, Santa Cruz, } \\
\text { California, USA }\end{array}$ & $4 \mathrm{~A} 4$ & $1 / 50$ & - & ++ & - & ++ \\
\hline ER & Novocastra & $6 \mathrm{~F} 11$ & $1 / 30$ & ++ & - & + & - \\
\hline PR & Dako & PgR 636 & $1 / 100$ & ++ & - & + & - \\
\hline Ki67 & Dako & MIB1 & $1 / 200$ & + & + & + & + \\
\hline c-kit (CD117) & Dako & 104D2 & $1 / 200$ & ++ & - & $+^{*}$ & - \\
\hline
\end{tabular}

Staining intensity: - , negative;,$+ 1-10 \% ;++,>10 \%$.

*Reactivity confined to entrapped luminal epithelial cells.

AME, adenomyoepithelioma; $\alpha$-SMA, $\alpha$ smooth muscle actin; CK, cytokeratin; CS, collagenous spherulosis; ER, oestrogen receptor; LECs, luminal epithelial cells; MECs, myoepithelial cells; PR, progesterone receptor; S-100, S-100 protein; SMMHC, smooth muscle myosin heavy chain. 


\section{Take home messages}

- We report the case of a 48 year old woman who presented with collagenous spherulosis of the breast in an adenomyoepithelioma

- The combination of these two benign lesions led to diagnostic difficulties; namely, differentiation from adenoid cystic carcinoma, but also cribriform carcinoma and cylindroma of the breast

- Immunohistochemistry using antibodies to the oestrogen receptor, progesterone receptor, p63, and c-kit (CD117) was useful in differentiating between these lesions

spheres, which were strongly reactive for collagen IV; and the lack of c-kit immunoreactivity. ${ }^{26-8}$

Invasive cribriform carcinoma of the breast is a special type of breast carcinoma with excellent prognosis. ${ }^{7}$ Unlike the other lesions, invasive cribriform carcinoma lacks the participation of myoepithelial cells, the presence of hyaline globules, basement membrane deposition, and is consistently positive for ER and PR. ${ }^{7}$

Cylindroma (dermal analogue tumour) is an exceedingly rare tumour of the mammary gland and only one case has been described so far. ${ }^{9}$ Histologically, the tumour showed nests of basophilic basaloid cells with darkly staining round nuclei, indistinct nucleoli, and scant cytoplasm, arranged in the typical "jigsaw" pattern seen in cutaneous cylindromas. Tumour cells were positive for CK7 and AE1/AE3, and focally reactive for $\alpha$ smooth muscle actin at the periphery of cell islands. ${ }^{9}$ S-100 protein and CDIA highlighted the presence of Langerhans cells within the cell islands. ${ }^{9}$ Collagen IV strongly stained hyaline globules and the basement membrane. Carcinoembryonic antigen, gross cystic fluid protein 15, ER, and PR were negative. Breast cylindroma and collagenous spherulosis share the presence of periodic acid Schiff D/ collagen IV positive basement membranes and "hyaline globules" lined by basaloid cells. ${ }^{9}$ However, in contrast to cylindroma, CS shows a different immunohistochemical profile and lacks Langerhans cells admixed with the epimyoepithelial cell layers. ${ }^{9}$

In conclusion, we report a case of breast AME with CS. Pathologists should be aware of the possible coexistence of these two lesions, to avoid a misdiagnosis of AdCC. In this instance, ER and PR, in addition to p63 and c-kit (CD117), proved to be useful adjunct antibodies to differentiate between these lesions.

\section{ACKNOWLEDGEMENTS}

$\mathrm{J}$ S Reis-Filho is the recipient of the Gordon Signy International Fellowship Award and is partially supported by a PhD grant from the Fundação para a Ciência e a Tecnologia (FCT), Ref.: SFRH/BD/5386/ 2001, Portugal. L G Fulford is an MD student supported by The Ludwig Institute for Cancer Research, London, UK.

\section{Authors' affiliations}

J S Reis-Filho, L G Fulford, S R Lakhani, The Breakthrough Toby Robins Breast Cancer Research Centre, Institute of Cancer Research, London SW3 6JB UK, UK

B Crebassa, Cabinet Prado Pathologie, Marseille 13008, France

S Carpentier, Marseille North University, Marseille 13385, France Correspondence to: Professor S R Lakhani, The Breakthrough Toby
Robins Breast Cancer Research Centre, Institute of Cancer Research, Mary-Jean Mitchell Green Building, Chester Beatty Laboratories, Fulham Road, London SW3 6JB, UK; Sunil.lakhani@icr.ac.uk

Accepted for publication 10 July 2003

\section{REFERENCES}

1 Hamperl H. The myothelia (myoepithelial cells). Normal state; regressive changes; hyperplasia; tumors. Curr Top Pathol 1970;53:161-220.

2 Tavassoli FA. Myoepithelial lesions of the breast. Myoepitheliosis, adenomyoepithelioma, and myoepithelial carcinoma. Am J Surg Pathol 1991; 15:554-68.

3 Rosen PP. Adenomyoepithelioma of the breast. Hum Pathol 1987; 18:1232-7.

4 Clement PB, Young RH, Azzopardi JG. Collagenous spherulosis of the breast. Am J Surg Pathol 1987;11:411-17.

5 Mooney EE, Kayani N, Tavassoli FA. Spherulosis of the breast. A spectrum of mucinous and collagenous lesions. Arch Pathol Lab Med 1999;123:626-30.

6 Kleer CG, Oberman HA. Adenoid cystic carcinoma of the breast: value of histologic grading and proliferative activity. Am J Surg Pathol 1998;22:569-75.

7 Trendell-Smith NJ, Peston D, Shousha S. Adenoid cystic carcinoma of the breast: a tumour commonly devoid of oestrogen receptors and related proteins. Histopathology 1999;35:241-8.

8 Van Dorpe J, De Pauw A, Moerman P. Adenoid cystic carcinoma arising in an adenomyoepithelioma of the breast. Virchows Arch 1998;432:119-22.

9 Gokaslan ST, Carlile B, Dudak M, et al. Solitary cylindroma (dermal analog tumor) of the breast: a previously undescribed neoplasm at this site. Am J Surg Pathol $2001 ; 25: 823-6$.

10 Holst VA, Marshall CE, Moskaluk CA, et al. KIT protein expression and analysis of c-kit gene mutation in adenoid cystic carcinoma. Mod Pathol $1999 ; 12: 956-60$. 